\title{
A prospective international study on adherence to treatment in 305 patients with flaring SLE: assessment by drug levels and by self-administered questionnaires
}

\begin{abstract}
Nathalie Costedoat-Chalumeau ${ }^{1}$, Frédéric Houssiau ${ }^{2}$, Peter Izmirly ${ }^{3}$, Véronique Le Guern ${ }^{1}$, Sandra Navarra ${ }^{4}$, Meenakshi Jolly ${ }^{5}$, Guillermo Ruiz-Irastorza ${ }^{6}$, Gabriel Baron ${ }^{7}$, Eric Hachulla $^{8}$, Nancy Agmon-Levin ${ }^{9}$, Yehuda Shoenfeld ${ }^{9}$, Francesca Dall'Ara ${ }^{10}$, Jill Buyon ${ }^{3}$, Christophe Deligny ${ }^{11}$, Ricard Cervera ${ }^{12}$, Estibaliz Lazaro ${ }^{13}$, Holy Bezanahary ${ }^{14}$, Gaëlle Leroux $^{15}$, Nathalie Morel ${ }^{1}$, Jean-François Viallard ${ }^{13}$, Christian Pineau ${ }^{16}$, Lionel Galicier ${ }^{17}$, Ronald Van Vollenhoven ${ }^{18}$, Angela Tincani ${ }^{10}$, Hanh Nguyen ${ }^{19}$, Guillaume Gondran ${ }^{14}$, Noel Zahr $^{20}$, Jacques Pouchot ${ }^{21}$, Jean-Charles Piette ${ }^{15}$, Michelle Petri ${ }^{*}{ }^{* 2}$, and David Isenberg ${ }^{*}, 19$ ${ }^{1}$ AP-HP, Cochin Hospital, Internal Medicine Department, Centre de référence maladies autoimmunes et systémiques rares, Paris, France; Université Paris Descartes-Sorbonne Paris Cité, Paris, France; INSERM U 1153, Center for Epidemiology and Statistics Sorbonne Paris Cité CRESS, Paris, France
\end{abstract}

${ }^{2}$ Service de Rhumatologie, Cliniques Universitaires Saint-Luc ; Pôle de Pathologies Rhumatismales Inflammatoires et Systémiques, Université catholique de Louvain, Brussels, Belgium

${ }^{3}$ Division of Rheumatology, Department of Medicine, New York University School of Medicine, New York, USA

${ }^{4}$ University of Santo Tomas Hospital, Rheumatology Center, Manila, Philippines

${ }^{5}$ Rush University Medical Center, Rush Lupus Clinic, Chicago, II, USA

${ }^{6}$ Autoimmune Diseases Research Unit, Department of Internal Medicine, BioCruces Health Research Institute, Hospital Universitario Cruces, University of the Basque Country, Barakaldo, Spain

${ }^{7}$ AP-HP, Hôpital Hôtel-Dieu, Centre d'Epidémiologie Clinique, Paris; Université Paris DescartesSorbonne Paris Cité, Paris, France

${ }^{8}$ Claude Huriez Hospital, Internal Medicine Department, Centre de Référence Maladies Autoimmunes et Systémiques rares, Université de Lille, Lille, France

${ }^{9}$ Sheba Medical Center, Zabludowicz Center for Autoimmune Diseases, Tel-Hashomer, Israel

\footnotetext{
Correspondence to: Prof. Nathalie Costedoat-Chalumeau, Hôpital Cochin, 75014, Paris, France. Fax number: +33158413246 . Phone number: +33687508123 nathalie.costedoat@gmail.com.

These authors contributed equally.

Conflict of interest: none

Author Contributions:

N.C.-C. wrote the manuscript; N.C.-C., J.-C.P., M.P., and D.I. designed the research; N.C.-C., F.H., P.I., V.L., S.N., M.J., G.R.-I., G.B., E.H., N.A.-L., Y.S., F.D., J.B., C.D., R.C., E.L., H.B., G.L., N.M., J.-F.V., C.P., L.G., R.v.V., A.T., H.N., G.G., J.P., J.-C.P., M.P., and

D.I. performed the research; N.C.-C. and G.B. analyzed the data; N.Z. contributed new reagents/analytical tools.
} 
${ }^{10}$ Rheumatology and Clinical Immunology Unit, Spedali Civili and Dpt. of Clinical and Experimental Science-University of Brescia, Italy

${ }^{11}$ Pierre-Zobda-Quitman Hospital, Internal Medicine Department, Martinique, France

${ }^{12}$ Hospital Clínic de Barcelona, Department of Autoimmune Diseases, Barcelona, Spain

${ }^{13}$ Haut Lévêque Hospital, Internal Medicine Department, Pessac, France

${ }^{14}$ Dupuytren Hospital, Internal Medicine Department, Limoges, France

${ }^{15}$ AP-HP, Pitié-Salpêtrière Hospital, Internal Medicine Department, Paris, France

${ }^{16}$ Montreal General Hospital, Lupus Clinic, Montreal, Canada

${ }^{17}$ AP-HP, St Louis Hospital, Clinical Immunology Department, Paris, France

${ }^{18}$ The Karolinska University Hospital, Department of Medicine, Unit for Clinical Research Therapy, Inflammatory Diseases, Stockholm, Sweden

${ }^{19}$ University College London, Centre for Rheumatology, London, UK

${ }^{20}$ AP-HP, Hopital Pitié-Salpêtrière, Pharmacology Department, Paris, France

${ }^{21}$ European Georges Pompidou Hospital, Internal Medicine Department, Paris, France

22Johns Hopkins University School of Medicine, Baltimore, MD, USA

\section{Abstract}

Nonadherence to treatment is a major cause of lupus flares. Hydroxychloroquine (HCQ), a major medication in systemic lupus erythematosus, has a long half-life and can be quantified by HPLC.

This international study evaluated nonadherence in 305 lupus patients with flares using drug levels (HCQ $<200 \mathrm{ng} / \mathrm{ml}$ or undetectable desethylchloroquine), and self-administered questionnaires (MASRI $<80 \%$ or MMAS- $8<6$ ).

Drug levels defined $18.4 \%$ of the patients as severely nonadherent. In multivariate analyses, younger age, non-use of steroids, higher BMI and unemployment were associated with nonadherence by drug level. Questionnaires classified 39.9\% of patients as nonadherent. Correlations between adherence measured by questionnaires, drug level, and physician assessment were moderate.

Both methods probably measured two different patterns of nonadherence: self-administered questionnaires mostly captured relatively infrequently missed tablets, while drug levels identified severe nonadherence (i.e., interruption or erratic tablet intake). The frequency with which physicians miss nonadherence, together with under-reporting by patients, suggests that therapeutic drug monitoring is useful in this setting.

Trial registration: ClinicalTrials.gov: NCT01509989

\section{Keywords}

Hydroxychloroquine; blood concentration; blood levels; systemic lupus erythematosus; adherence; desethylchloroquine 
Therapeutic management of systemic lupus erythematosus (SLE), a systemic autoimmune disorder with significant morbidity and mortality, may include nonsteroidal antiinflammatory drugs, hydroxychloroquine (HCQ), low to high doses of corticosteroids, and immunosuppressive agents, as well as biotherapies. ${ }^{1}$ As in many other chronic diseases, the effectiveness of these treatments (at least for self-administered medication) is impaired by nonadherence to treatment, reported to vary between 3\% and 76\% in SLE depending on the study and assessment method used, and all have limitations. ${ }^{2}$

Hydroxychloroquine has been recognized as the cornerstone of SLE treatment; among its other beneficial effects it reduces SLE flares. ${ }^{3-7}$ HCQ is rapidly absorbed after oral administration (2-4 h) and is relatively unaffected by concomitant food. HCQ protein binding is about $50 \%$ to both albumin and alpha glycoprotein. The volume of distribution is very large due to extensive sequestration of the drug by tissues. ${ }^{4,8}$ From 21 to $70 \%$ of HCQ is excreted without metabolism while the rest is mostly metabolized via cytochrome P450 2D6 (the main isoform involved in a population of Korean lupus patients), ${ }^{9} 3 \mathrm{~A} 4,3 \mathrm{~A} 5$, and $2 \mathrm{C} 8$ isoforms. ${ }^{9}$ Approximately half of unchanged HCQ and metabolites are excreted through the kidneys. ${ }^{4}, 10$

HCQ and desethylchloroquine (DCQ), its main metabolite, can be quantified by high performance liquid chromatography (HPLC). A pharmacokinetic/pharmacodynamic (PK/PD) relation for HCQ has been found in rheumatoid arthritis, ${ }^{11,} 12$ cutaneous lupus, ${ }^{13}$ and SLE; a low blood HCQ level is a marker and predictor of SLE exacerbation. ${ }^{14}$ Although the large French multicenter randomized prospective study PLUS did not confirm the interest of adapting daily HCQ dosage to its blood level in terms of efficacy, it did confirm the correlation between HCQ levels and efficacy. ${ }^{15}$ Possibly most importantly, since the blood half-life of HCQ is at least 5 days, and the terminal half-life of HCQ is 43 days, ${ }^{8}$ very low blood HCQ levels objectively indicate severe nonadherence (i.e., identify patients who have not taken HCQ for a significant period and not those who miss a few tablets). ${ }^{2}, 16-24$

Here, we studied the frequency and determinants of nonadherence in SLE flaring patients treated with HCQ by assessing nonadherence by 2 different methods: blood drug assays (HCQ and DCQ levels), and patient questionnaires (Medication Adherence Self-Report Inventory scale (MASRI) and the Morisky Medication Adherence Scale (MMAS-8)). We correlated the results of these two methods with physician evaluations of nonadherence.

\section{RESULTS}

\section{Study population}

The study included 305 patients ( 288 women; mean ( \pm SD) age $37.7 \pm 11.6$ years): 304 had blood HCQ levels assessed at inclusion and 303 completed at least 1 adherence questionnaire. Ethnic groups included 153 whites (50.2\%), 83 blacks (27.2\%), and 69 others (22.6\%). The daily dose of HCQ was $400 \mathrm{mg}$ for 219 patients (71.8\%), $200 \mathrm{mg}$ for 47 (15.4\%), and another dose (always $>200 \mathrm{mg}$ ) for $39(12.8 \%)$.

Median [interquartile ranges: Q1-Q3] disease duration was 10.0 [5.0-15.0] years, and the median duration of HCQ treatment was 7.5 [3.6-12.1] years (1 missing value). All patients 
had positive tests for antinuclear antibodies, 109 (35.7\%) a history of renal involvement, and $32(10.5 \%)$ an associated antiphospholipid syndrome (1 missing value).

At inclusion, the median SLEDAI [Q1-Q3] was 8.0 [6.0-10.0]. According to the SELENASLEDAI composite flare index, flares were mild or moderate in 173 patients $(56.7 \%)$ and severe in $132(43.3 \%)$. Flares were renal in $67(22 \%)$, and neurological in only $3(1 \%)$. They resulted in the hospitalization of 74 patients $(24.3 \%)$ and an increased steroid dose for 210 $(68.9 \%)$.

\section{Nonadherence by drug levels}

Among the 304 patients with drug level measurements, mean blood HCQ was $812 \pm 618$ $\mathrm{ng} / \mathrm{ml}$, and mean blood DCQ $122 \pm 95 \mathrm{ng} / \mathrm{ml}$. HCQ levels were very low $(<200 \mathrm{ng} / \mathrm{ml})$ in 44 (14.5\%) patients, and undetectable in $22(7.2 \%)$. DCQ was undetectable in $48(15.8 \%)$ patients. Overall, $56(18.4 \%)$ patients were nonadherent, as defined by a blood HCQ level below $200 \mathrm{ng} / \mathrm{ml}$ and/or undetectable blood DCQ. The proportion of nonadherent patients by drug level was $15.2 \%$ (7/46) in patients with a prescribed HCQ dose of $200 \mathrm{mg} / \mathrm{d}$ and $18.3 \%$ (40/219) in those with a $400 \mathrm{mg} / \mathrm{d}$ dose.

Univariate analyses showed that the 56 patients classified as nonadherent by drug level differed from the other patients only for age at SLE diagnosis (23.1 \pm 8.9 versus $28.0 \pm 11.3$; $P=0.003$ ), frequency of unemployment (46.3\% (25/54) versus $30.9 \%$ (75/243), $P=0.030$ ), and prescription of a steroid treatment (60.7\% (34/56) versus $79.8 \%(198 / 248), P=0.002)$. Drug level assessment at inclusion was more often their first drug measurement $(83.9 \%$ (47/56) versus $70.9 \%$ (175/247), $P=0.046)$.

The multivariate analyses showed that younger age at diagnosis $(P<0.001$; OR per 5 years 0.71 [95\%CI: $0.59-0.85])$, non-use of steroids ( $P<0.001$; OR 3.73 [95\%CI: 1.84-7.56]), higher BMI $\left(P=0.017\right.$; OR per $5 \mathrm{~kg} / \mathrm{m}^{2} 1.35$ [95\%CI: 1.06-1.72]), and unemployment $(P=0.010$; OR 2.38 [95\%CI: 1.23-4.61])were associated with nonadherence by drug level (Table 2).

\section{Effect of previous blood HCQ measurement}

As part of their routine clinical management, $81 / 303$ patients (26.7\%) had undergone at least one blood HCQ measurement in the months or years preceding inclusion in the current study, and poor adherence had been diagnosed at least once for $36.8 \%$.

At inclusion, median HCQ levels of patients with a previous blood HCQ measurement was 839 [600-1140] versus 637 [316-1045] in those not previously tested. Nine of the 81 patients $(11.1 \%)$ with a previous HCQ measurement were nonadherent by drug level versus 47 of the $222(21.2 \%)$ never tested $(P=0.046)$.

\section{Nonadherence by self-administered questionnaires}

The MASRI score for HCQ averaged $85.8 \pm 20.5$ (6 missing values) and was $<80$ for $70 / 299$ patients (23.4\%); the MMAS-8 score for HCQ averaged 6.1 2.0 (4 missing values) and was $<6$ for 112/301 patients (37.2\%). Accordingly, questionnaires classified 121/303 patients $(39.9 \%)$ as nonadherent. 
In the univariate analyses (Table 1), the 121 patients classified as nonadherent by questionnaires differed from the other patients for age at inclusion (34.7 \pm 10.0 versus $39.7 \pm 12.2 ; P<0.001)$ and at SLE diagnosis $(24.2 \pm 8.8$ vs $29.0 \pm 12.0 ; P=0.001)$, race (white: $37.2 \%$ [45/121] vs $59.3 \%$ [108/182]; black: $34.7 \%$ [42/121] vs $22.5 \%$ [41/182]; and other ethnicity: $28.1 \%$ [34/121] vs $18.1 \%$ [33/182], $P=0.0008$ ) and frequency of steroid treatment (68.6\% [83/121] vs $81.3 \%$ [148/182], $P=0.011)$. Current hospitalization in nonadherent patients was $29.8 \%(36 / 121)$ versus $20.3 \%(37 / 182)$ in the others $(P=0.060)$. We note that $70.6 \%$ of whites self-reported that they were adherent versus $49.4 \%$ of blacks and $49.3 \%$ of others $(P=0.0008)$.

According to the multivariate analyses, only younger age at diagnosis $(P<0.001$; OR per 5 years 0.79 [95\%CI: 0.70-0.90]), race (black versus white: $\mathrm{p}=0.015$; OR 2.03 [95\%CI: $1.15-$ 3.61], and another ethnicity versus white: $\mathrm{p}=0.012$; OR 2.19 [95\%CI: 1.19-4.06]), and nonuse of steroids ( $P=0.005$; OR 2.29 [95\%CI: $1.28-4.10]$ ) were associated with nonadherence by questionnaire (Table 3 ).

\section{Overall nonadherence}

In all, 137/302 patients (45.4\%) were considered nonadherent to HCQ treatment by at least one criterion (drug levels or questionnaires), and 40/302 (13.2\%) by both methods. Interestingly, 24 (43\%) of the nonadherent patients by blood drug level (including patients with both HCQ and DCQ undetectable) would have been classified as adherent based on at least one of the two questionnaires.

\section{Physicians' assessment of nonadherence}

Median adherence [Q1-Q3] to HCQ treatment in the previous month was evaluated by physicians at 85 [68-94] on a visual analog scale (VAS) from 0 (complete nonadherence) to 100 (full adherence). Physicians estimated that $123 / 305$ patients (40.3\%) took less than $80 \%$ of their HCQ treatment in the previous month and that only 12/305 (3.9\%) took less than $20 \%$ (Figure 1).

Among the 56 patients nonadherent by drug level, physician's median adherence assessment was 75.1 [42.7-89.6] vs 87.0 [70.0-95.1] for the other patients ( $P<0.0001)$. Physicians estimated that $32 / 56(57.1 \%)$ of the patients nonadherent by drug level took less than $80 \%$ of their treatment and that only $8 / 56(14.3 \%)$ took less than $20 \%$.

\section{Correlation between adherence by drug level, by questionnaire, and by physician assessment}

The correlation between adherence by drug level and by questionnaire was moderate (Table 4), with a Spearman rank correlation $\left(r_{s}\right)$ of 0.37 for the MASRI HCQ and of 0.31 for the MMAS-8 HCQ. Correlation between adherence by questionnaire and physician assessment was also moderate with an $\mathrm{r}_{\mathrm{s}}$ of 0.43 for the MASRI HCQ and 0.36 for the MMAS-8 HCQ. The correlation between adherence by drug level and physician assessment was weaker, with an $r_{s}$ of 0.19 and even worse when patients with a previous blood HCQ measurement were excluded $\left(r_{s}=0.16\right)$. This did not affect the other correlations, however. 


\section{Nonadherence to prednisone and immunosuppressive drugs}

In addition to HCQ, 233/305 patients (76.4\%) were treated with prednisone, and 141/305 patients (46.2\%) with immunosuppressive drugs or biotherapies at inclusion. Physicians evaluated median adherence [Q1-Q3] to HCQ and steroids in the previous month at 84.6 [68.0-94.3] and 90.3 [79.1-97.1] respectively.Table 4 shows a strong correlation between evaluation of nonadherence to HCQ and evaluation of nonadherence to other SLE medications, regardless of mode of evaluation.

\section{DISCUSSION}

This prospective international study of flaring SLE patients showed nonadherence rates to HCQ treatment of $18.4 \%$ by drug level, $39.9 \%$ by questionnaire, and $44.9 \%$ overall. Nonadherence to other SLE treatments (especially steroids) could not be objectively measured but self-reported nonadherence to HCQ and to other treatments as well as physician-estimated adherence to HCQ and to steroids correlated well.

Evaluating nonadherence is difficult: methods vary widely and do not capture the same patterns of nonadherence. ${ }^{2,16,18,20,25-41}$ Studies using self-administered questionnaires report that $17-30 \%$, 40 of patients take less than $80 \%$ of their treatment. Consistently, only $23.4 \%$ of our patients admitted HCQ adherence below $80 \%$ with the MASRI. Given that $<80 \%$ is a high threshold for nonadherence, such small numbers (for the MASRI) are very encouraging. Nonetheless, these questionnaires, based on what patients are willing to admit, are not objective and underestimate nonadherence. ${ }^{2}$ More objective methods report strikingly higher nonadherence rates. Using electronic monitoring over a two-year period, Marengo et al. showed that $76 \%$ of the 78 participating patients had an adherence rate below $80 \% .{ }^{35}$ In a recent large study using pharmacy refill information from US Medicaid data, Feldman et al. found that $79 \%$ of 9,600 new users of HCQ had an adherence rate $<80 \%$. ${ }^{42}$ These objective nonadherence rates are much higher than the $23.4 \%$ self-reported nonadherence rate and the $40.3 \%$ physician-estimated nonadherence rate in our study, which are probably highly optimistic, especially as we assessed flaring patients, known to be at higher risk of nonadherence. ${ }^{42}$

Few studies have assessed adherence by blood HCQ measurements. We previously reported that 14 of 203 patients (7\%) had HCQ levels lower than $200 \mathrm{ng} / \mathrm{ml}$ and thereafter admitted severe nonadherence. ${ }^{20}$ Ting et al., defining nonadherence by a blood HCQ level less than $100 \mathrm{ng} / \mathrm{ml}$, found that 12 of 41 adolescents and young adults with SLE (29\%) were nonadherent. ${ }^{16}$ Moreover, adherence estimated with blood HCQ correlated well with that measured by pharmacy refill information. ${ }^{16}$ Recently, Ludici et al, using the same cut-off of $100 \mathrm{ng} / \mathrm{ml}$, found that 24 of 83 SLE patients (29\%) in remission were nonadherent. ${ }^{24}$ Using a level below $15 \mathrm{n} \mathrm{g} / \mathrm{ml}$, Durcan et al. found that 88 of 686 patients (13\%) were severely nonadherent. ${ }^{21}$ Here, we found a nonadherence rate of $14.5 \%$ with HCQ levels only and $18.4 \%$ when we also considered undetectable DCQ levels. These percentages are in keeping with the $5-10 \%$ of patients who completely stopped or frequently interrupted tablet ingestion in studies using electronic monitoring. ${ }^{43,} 44$ Blood drug level measurements can objectively detect only severe nonadherence (i.e. absence of any treatment or intake of only a few tablets), since high HCQ levels may be reached within days after treatment resumes ${ }^{19}$ 
due especially to flares or simply the 'white-coat' compliance effect. ${ }^{2}$ Accordingly, rates of nonadherence by drug levels are likely to reveal only the tip of the iceberg. It is nonetheless worth knowing, both because of its deleterious consequences, and because physicians are frequently unaware of it.

The lack of overlap between questionnaires and drug levels, which probably measure different patterns of nonadherence, is reflected by the moderate correlation between these methods. Thus, two separate and independent patterns of nonadherence have been described: 43 (a) tablets missed relatively infrequently (but more than 20\%) and (b) tablet intake completely stopped or frequently interrupted and erratic. Interestingly, some patients with undetectable levels of both HCQ and DCQ (i.e., who had not taken any treatment for some time) had MASRI scores greater than $80 \%$. This observation suggests that some patients are very reluctant to admit severe nonadherence, perhaps even to themselves. We may hypothesize that questionnaires can capture the first pattern, while very low blood drug levels identify only the latter. These methods may thus be regarded as complementary.

The factors associated with nonadherence by drug level by multivariate analyses were age (younger) at diagnosis, non-use of steroids, BMI, and unemployment whereas those associated with nonadherence by questionnaire were age at diagnosis, steroid non-use, and race (black or another ethnicity, versus white). The absence of steroid prescriptions may reflect milder disease, whereas younger age at diagnosis, BMI, and unemployment are standard factors of nonadherence. Nonwhite patients reported nonadherence by questionnaire more frequently, but drug levels did not differentiate severe nonadherence according to ethnicity.

Improving treatment adherence is very difficult. Studies have used different methods, often complex and time-consuming, but have found inconstant and only small effects on adherence. ${ }^{2}$ By contrast, Durcan et al. showed in a large cohort that routine measurement of HCQ levels usually led to adherence improvement over time. Our study confirms the potential value of repeated assays to improve adherence: patients with at least one previous HCQ measurement were less likely to be nonadherent by drug level. Our experience is that physicians are sometimes very surprised to discover nonadherence by drug level, particularly in patients who never miss medical appointments and who regularly perform ophthalmological tests to detect HCQ toxicity. Consistently, we found a poor correlation between nonadherence by drug level and by physician assessment. This is not surprising as clinical judgment of adherence has been found wanting in almost every relevant study. ${ }^{45} \mathrm{~A}$ more pessimistic interpretation of our result (and those of Durcan et al., ${ }^{21}$ ) however, is that some patients may briefly improve HCQ adherence because they suspect they might be tested at their next clinical visit. ${ }^{19}$ In this setting, undetectable levels of DCQ might be particularly interesting since unmasking nonadherence is the first step in trying to improve it.

Our study has some limitations. First, there is no gold standard method for measuring nonadherence. We used a previously validated HCQ cut-off, ${ }^{20}$ but other cut-offs could have been used. ${ }^{16,21,24}$ Although our clinical experience since our first publication ${ }^{20}$ confirms the clinical relevance of this cut-off $(200 \mathrm{ng} / \mathrm{ml})$, no large study has conducted retrospective 
interviews of patients to confirm nonadherence. Second, multiple corticosteroids have been found to induce higher levels of expression of the CYP (2D6) that metabolizes HCQ. ${ }^{46}$ The high doses of corticosteroids that flaring lupus patients may receive could serve as a confounding factor that lowers their HCQ levels and thus results in overestimation of nonadherence by therapeutic drug monitoring. However, CYP metabolism is not the major route of elimination for HCQ; the 2D6 isoform may not be the main isoform involved in non-Korean populations; the drug is sequestered in tissues; our definition used for severe nonadherence was relatively stringent, and most of our patients had undetectable DCQ levels. These facts make it unlikely that this mechanism could have significantly altered our results. Third, although it is logical from a pharmacological perspective to look for undetectable DCQ levels, its use requires further validation. Fourth, we were unable to objectively assess nonadherence to steroids and immunosuppressive drugs by assays, because of their much shorter half-life. Furthermore, our inclusion of only flaring patients precludes the generalization of our figures to all lupus cohorts: flares could have led to an overestimation of nonadherence given the inverse association between adherence and lupus activity, but also to an underestimation since patients might have resumed their treatment between the onset of the flare and the inclusion in the study. Similarly, since some of our centers routinely use drug levels to assess adherence (a quarter of our patients had previously been assessed for HCQ level, before inclusion), nonadherence might have been underestimated. Finally, because this study took place mainly in expert SLE centers and was aimed at detecting nonadherence, participation necessarily increased clinicians' awareness of this issue. In real life, the poor correlation between physician evaluation and confirmed nonadherence might be even worse.

In conclusion, almost half of patients were nonadherent and $20 \%$ were objectively severely nonadherent, often without their physicians' knowledge. Questionnaires and drug levels captured different patterns of nonadherence and seem complementary. Questionnaires are simple and inexpensive but underestimate nonadherence and are rarely used for practical reasons. Blood drug measurements are easier to implement in routine clinical setting, but detect only severe nonadherence. Finally, given the pivotal importance of keeping steroid use as low as possible in SLE patients ${ }^{6}$ and considering that the steroid dose had to be increased in $68.9 \%$ of these flaring patients, unmasking nonadherence in this subset of patients is really useful, for it may avoid unnecessary treatment escalation. We thus recommend routinely measuring HCQ drug levels and using questionnaires as tools to assess nonadherence in SLE patients on HCQ.

\section{PATIENTS AND METHODS}

\section{Patients}

The study was an international, prospective, observational multicenter study conducted from January 2013 through June 2015 in 19 centers in 10 countries. Patients were included if they fulfilled the following inclusion criteria (1) diagnosis of SLE according to the SLICC classification criteria, ${ }^{47}$ (2) HCQ treatment for at least 2 months with a stable daily HCQ dosage of at least $200 \mathrm{mg}$, and (3) SLE flare, as defined by the SELENA-SLEDAI flare composite score. ${ }^{48}$ This score defines mild-to-moderate and severe flares and includes 3 
elements: the SELENA-SLEDAI score; an assessment of new or worsening disease activity, medication changes, and hospitalizations not captured by the SLEDAI alone; and physician's global assessment (PGA) on a VAS (from 0 to 3 ). ${ }^{48}$

Patients were excluded if they were unable to take oral medications or had received chloroquine in the past 2 months (because it interferes with blood HCQ measurements).

At inclusion, patients underwent a complete physical examination and local laboratory testing including a complement assay and anti-double-stranded DNA antibody assays. Whole-blood HCQ and DCQ levels were measured in a centralized laboratory (Paris, France) by HPLC with fluorometric detection. ${ }^{14}$ The patient's physician scored all components of the flare composite index and estimated adherence to HCQ treatment (and to other SLE treatments when relevant) in the past month on a VAS ranging from 0 (complete nonadherence) to 100 (full adherence). Patients completed 3 self-administered questionnaires: 2 dealing with treatment adherence (described below), and the Hospital Anxiety and Depression Scale (HADS) ${ }^{49}$ which measures anxiety and depression. ${ }^{49}$ It is a 14-item scale (7 relating to anxiety and 7 to depression) with a 4-point (0-3) response category, so that both anxiety and depression scores can range from 0 to 21 . A score of 0 to 7 for each subscale is interpreted as within the normal range, a score of 11 or higher as indicating a probable mood disorder, and a score of 8 to 10 as suggestive of it. ${ }^{50}$

\section{Evaluation and definition of nonadherence}

Adherence was evaluated by drug levels and by 2 questionnaires: part A of the Medication Adherence Self-Report Inventory scale (MASRI) ${ }^{33}$ and the Morisky Medication Adherence Scale (MMAS-8). Patients treated with other SLE medication completed separate MASRI and MMAS-8 forms for "other SLE medication," which included (in the same questionnaires) steroids, immunosuppressive drugs, and biotherapies.

The MASRI is a self-administered questionnaire assessing adherence from the patient's point of view. A MASRI $\geq 80 \%$ is considered good adherence to treatment. Part A consists of five 4-point scale items and one VAS item. The latter asks patients how much medication they have taken in the past month on a scale from $0 \%$ to $100 \%$ and is the only item used to estimate adherence quantitatively. The other 5 items simply assist patients in estimating their adherence. The MMAS-8 is an 8-item questionnaire that produces scores ranging from 0 to 8; higher scores indicate higher adherence.

Nonadherence was defined in 3 ways: by drug levels, by questionnaires, and by overall nonadherence. Nonadherence by drug level was defined by blood HCQ level $<200 \mathrm{ng} / \mathrm{ml}$ as previously validated, or by undetectable blood DCQ, indicating either the complete absence of HCQ treatment (when HCQ levels are also undetectable) or very recent treatment resumption (when very low HCQ levels are detected). Nonadherence by questionnaires was defined as MASRI $<80 \%$ and/or MMAS- $8<6$. Overall nonadherence was defined as nonadherence by either criterion. 


\section{Study oversight}

The study was conducted in accordance with the International Conference on Harmonization Guidelines for Good Clinical Practice and the ethical principles of the Declaration of Helsinki. A French ethics committee (Saint-Louis Hospital) and the local institutional review boards for each center approved the study protocol. All participants provided written informed consent.

\section{Statistical analysis}

Qualitative variables are described by proportions and percentages, and quantitative variables by means and standard deviations (SD) or medians and interquartile ranges (Q1Q3). The Chi-square test (or Fisher's test, as appropriate) and Student's t-test (or the MannWhitney U test) tested differences between nonadherent and other patients. Multiple logistic regressions were used to identify the variables independently associated with nonadherence. Every variable with $P<0.15$ by univariate analysis was included in the multiple-variable regression model, except for age to avoid multicollinearity between age and age at diagnosis. Similarly, because creatinine was not associated with nonadherence by drug level, creatinine clearance was excluded to avoid multicollinearity with body mass index (BMI). We performed stepwise selection after performing 1000 bootstrap re-samplings to assess the consistency of variable selection across randomly resampled data sets. Variables that appeared in at least $60 \%$ of the models were retained. Results are expressed as odds ratios with their $95 \%$ confidence intervals $(95 \% \mathrm{CI})$. Spearman rank correlations were used to assess the relations between adherence by drug level, by questionnaire, and by physician assessment. Receiver operating characteristics (ROC) analysis was used to evaluate the ability of MASRI and MMAS-8 values to discriminate between adherence and nonadherence, with nonadherence by drug level as the reference standard. Youden's index [(sensitivity + specificity)- 1] was used to identify the cut-off points for MASRI and MMAS-8 that optimized sensitivity and specificity. Statistical significance was defined by $P<0.05$. The statistical analysis used SAS 9.3 (SAS Inst. Inc., Cary, NC, USA).

\section{Acknowledgments}

We would like to thank the URC-CIC Paris Descartes Necker/Cochin (Ms Séverine Poignad and Leyya Mansoor) for implementation, monitoring, and data management of the study as well as Ms Kubéraka Mariampillai, Séverine Nieuwland-Husson, and Ada Clarke for their precious assistance.

Funding: This study was funded by a research grant from the French Ministry of Health (PHRC 2012: n ○12-002-0114), and sponsored by the Département de la Recherche Clinique et du Développement de l'Assistance Publique-Hôpitaux de Paris. The Hopkins Lupus Cohort was funded by NIH AR 43727

\section{References}

1. Rahman A, Isenberg DA. Systemic lupus erythematosus. N Engl J Med. 2008; 358:929-939. [PubMed: 18305268]

2. Costedoat-Chalumeau N, et al. Adherence to treatment in systemic lupus erythematosus patients. Best Pract Res Clin Rheumatol. 2013; 27:329-340. [PubMed: 24238690]

3. Costedoat-Chalumeau N, Amoura Z, Hulot JS, Lechat P, Piette JC. Hydroxychloroquine in systemic lupus erythematosus. Lancet. 2007; 369:1257-1258. 
4. Costedoat-Chalumeau, N., Leroux, G., Piette, J-C., Amoura, Z. Antimalarials and systemic lupus erythematosus. In: Lahita, RG.Tsokos, G.Buyon, JP., Koike, T., editors. Systemic lupus erythematosus. 5. Elsevier; 2010. p. 1061-81.

5. Ruiz-Irastorza G, Ramos-Casals M, Brito-Zeron P, Khamashta MA. Clinical efficacy and side effects of antimalarials in systemic lupus erythematosus: a systematic review. Ann Rheum Dis. 2010; 69:20-28. [PubMed: 19103632]

6. van Vollenhoven RF, et al. Treat-to-target in systemic lupus erythematosus: recommendations from an international task force. Ann Rheum Dis. 2014; 73:958-967. [PubMed: 24739325]

7. Chasset F, Arnaud L, Costedoat-Chalumeau N, Zahr N, Bessis D, Frances C. The effect of increasing the dose of hydroxychloroquine (HCQ) in patients with refractory cutaneous lupus erythematosus (CLE): An open-label prospective pilot study. J Am Acad Dermatol. 2016; 74:693699. [PubMed: 26850655]

8. Carmichael SJ, Charles B, Tett SE. Population pharmacokinetics of hydroxychloroquine in patients with rheumatoid arthritis. Ther Drug Monit. 2003; 25:671-681. [PubMed: 14639053]

9. Lee JY, et al. Polymorphisms of Cytochrome P450 2D6 are associated with blood hydroxychloroquine levels in patients with systemic lupus erythematosus. Arthritis Rheumatol. 2016; 68:184-190. [PubMed: 26316040]

10. Rainsford KD, Parke AL, Clifford-Rashotte M, Kean WF. Therapy and pharmacological properties of hydroxychloroquine and chloroquine in treatment of systemic lupus erythematosus, rheumatoid arthritis and related diseases. Inflammopharmacology. 2015; 23:231-269. [PubMed: 26246395]

11. Munster T, et al. Hydroxychloroquine concentration-response relationships in patients with rheumatoid arthritis. Arthritis Rheum. 2002; 46:1460-1469. [PubMed: 12115175]

12. Tett SE, Cutler DJ, Beck C, Day RO. Concentration-effect relationship of hydroxychloroquine in patients with rheumatoid arthritis--a prospective, dose ranging study. J Rheumatol. 2000; 27:16561660. [PubMed: 10914847]

13. Frances $\mathrm{C}$, et al. Low blood concentration of hydroxychloroquine in patients with refractory cutaneous lupus erythematosus: a French multicenter prospective study. Arch Dermatol. 2012; 148:479-484. [PubMed: 22508872]

14. Costedoat-Chalumeau N, et al. Low blood concentration of hydroxychloroquine is a marker for and predictor of disease exacerbations in patients with systemic lupus erythematosus. Arthritis Rheum. 2006; 54:3284-3290. [PubMed: 17009263]

15. Costedoat-Chalumeau N, et al. Hydroxychloroquine in systemic lupus erythematosus: results of a French multicentre controlled trial (PLUS Study). Ann Rheum Dis. 2013; 72:1786-1792. [PubMed: 23144449]

16. Ting TV, et al. Usefulness of cellular text messaging for improving adherence among adolescents and young adults with systemic lupus erythematosus. J Rheumatol. 2012; 39:174-179. [PubMed: 22089460]

17. Petri M, Fand H, Clarke W. Hydroxychloroquine levels identify four distinct subsets of systemic lupus erythematosus patients (abstract). Arthritis Rheum. 2013; 65:S770.

18. Lee JY, Luc S, Greenblatt DJ, Kalish R, McAlindon TE. Factors associated with blood hydroxychloroquine level in lupus patients: renal function could be important. Lupus. 2013; 22:541-542. [PubMed: 23396569]

19. Jallouli M, et al. Determinants of Hydroxychloroquine Blood Concentration Variations in Systemic Lupus Erythematosus. Arthritis Rheumatol. 2015; 67:2176-2184. [PubMed: 25989906]

20. Costedoat-Chalumeau N, et al. Very low blood Hydroxychloroquine concentrations as an objective marker of poor adherence to treatment in systemic lupus erythematosus. Ann Rheum Dis. 2007; 66:821-824. [PubMed: 17324970]

21. Durcan L, Clarke WA, Magder LS, Petri M. Hydroxychloroquine Blood Levels in Systemic Lupus Erythematosus: Clarifying Dosing Controversies and Improving Adherence. J Rheumatol. 2015; 42:2092-2097. [PubMed: 26428205]

22. Croyle L, Morand EF. Optimizing the use of existing therapies in lupus. Int J Rheum Dis. 2015; 18:129-137. [PubMed: 25884457] 
23. Lee JY, Lee J, Kwok SK, Ju JH, Park KS, Park SH. Factors related to blood hydroxychloroquine concentration in patients with systemic lupus erythematosus. Arthritis Care Res (Hoboken). 2016 [Epub ahead of print].

24. Iudici, M., et al. Health status and concomitant prescription of immunosuppressants are risk factors for hydroxychloroquine non-adherence in systemic lupus patients with prolonged inactive disease. Lupus. 961203317717631. 2017.

25. Petri M, Perez-Gutthann S, Longenecker JC, Hochberg M. Morbidity of systemic lupus erythematosus: role of race and socioeconomic status. Am J Med. 1991; 91:345-353. [PubMed: 1951378]

26. Uribe AG, et al. Systemic lupus erythematosus in three ethnic groups. XVIII. Factors predictive of poor compliance with study visits. Arthritis Rheum. 2004; 51:258-263. [PubMed: 15077269]

27. Mosley-Williams A, Lumley MA, Gillis M, Leisen J, Guice D. Barriers to treatment adherence among African American and white women with systemic lupus erythematosus. Arthritis Rheum. 2002; 47:630-638. [PubMed: 12522837]

28. Mirotznik J, Ginzler E, Zagon G, Baptiste A. Using the health belief model to explain clinic appointment-keeping for the management of a chronic disease condition. J Community Health. 1998; 23:195-210. [PubMed: 9615295]

29. Gladman DD, Koh DR, Urowitz MB, Farewell VT. Lost-to-follow-up study in systemic lupus erythematosus (SLE). Lupus. 2000; 9:363-367. [PubMed: 10878729]

30. Rojas-Serrano J, Cardiel MH. Lupus patients in an emergency unit. Causes of consultation, hospitalization and outcome. A cohort study. Lupus. 2000; 9:601-606. [PubMed: 11035435]

31. Oliveira-Santos M, Verani JF, Klumb EM, Albuquerque EM. Evaluation of adherence to drug treatment in patients with systemic lupus erythematosus in Brazil. Lupus. 2011; 20:320-329. [PubMed: 21183558]

32. Duvdevany I, Cohen M, Minsker-Valtzer A, Lorber M. Psychological correlates of adherence to self-care, disease activity and functioning in persons with systemic lupus erythematosus. Lupus. 2011; 20:14-22. [PubMed: 20837569]

33. Koneru S, et al. Effectively measuring adherence to medications for systemic lupus erythematosus in a clinical setting. Arthritis Rheum. 2007; 57:1000-1006. [PubMed: 17665465]

34. Chambers SA, Raine R, Rahman A, Isenberg D. Why do patients with systemic lupus erythematosus take or fail to take their prescribed medications? A qualitative study in a UK cohort. Rheumatology (Oxford). 2009; 48:266-271. [PubMed: 19151034]

35. Marengo MF, et al. Measuring therapeutic adherence in systemic lupus erythematosus with electronic monitoring. Lupus. 2012; 21:1158-1165. [PubMed: 22588588]

36. Bruce IN, Gladman DD, Urowitz MB. Factors associated with refractory renal disease in patients with systemic lupus erythematosus: the role of patient nonadherence. Arthritis Care Res. 2000; 13:406-408. [PubMed: 14635317]

37. Sailler L, et al. Blood concentrations of hydroxychloroquine and its desethyl derivative correlate negatively with the percentage of CD45RO+ cells among CD4+ lymphocytes in hydroxychloroquine-treated lupus patients. Ann N Y Acad Sci. 2007; 1108:41-50. [PubMed: 17893969]

38. Ward MM, Lotstein DS, Bush TM, Lambert RE, van Vollenhoven R, Neuwelt CM. Psychosocial correlates of morbidity in women with systemic lupus erythematosus. J Rheumatol. 1999; 26:2153-2158. [PubMed: 10529132]

39. Julian LJ, et al. Depression, medication adherence, and service utilization in systemic lupus erythematosus. Arthritis Rheum. 2009; 61:240-246. [PubMed: 19177526]

40. Daleboudt GM, Broadbent E, McQueen F, Kaptein AA. Intentional and unintentional treatment nonadherence in patients with systemic lupus erythematosus. Arthritis Care Res (Hoboken). 2011; 63:342-350. [PubMed: 21120967]

41. Garcia-Gonzalez A, et al. Treatment adherence in patients with rheumatoid arthritis and systemic lupus erythematosus. Clin Rheumatol. 2008; 27:883-889. [PubMed: 18185905]

42. Feldman CH, Yazdany J, Guan H, Solomon DH, Costenbader KH. Medication Nonadherence Is Associated With Increased Subsequent Acute Care Utilization Among Medicaid Beneficiaries 
With Systemic Lupus Erythematosus. Arthritis Care Res (Hoboken). 2015; 67:1712-1721. [PubMed: 26097166]

43. Rudd P, Lenert L. Pharmacokinetics as an aid to optimising compliance with medications. Clin Pharmacokinet. 1995; 28:1-6. [PubMed: 7712658]

44. Dusing R, Lottermoser K, Mengden T. Compliance with drug therapy-new answers to an old question. Nephrol Dial Transplant. 2001; 16:1317-1321. [PubMed: 11427617]

45. Osterberg L, Blaschke T. Drug Therapy: Adherence to Medication. N Engl J Med. 2005; 353:487497. [PubMed: 16079372]

46. Farooq M, Kelly EJ, Unadkat JD. CYP2D6 Is Inducible by Endogenous and Exogenous Corticosteroids. Drug metabolism and disposition: the biological fate of chemicals. 2016; 44:750 757. [PubMed: 26965986]

47. Petri M, et al. Derivation and validation of the Systemic Lupus International Collaborating Clinics classification criteria for systemic lupus erythematosus. Arthritis Rheum. 2012; 64:2677-2686. [PubMed: 22553077]

48. Petri M, et al. Combined oral contraceptives in women with systemic lupus erythematosus. N Engl J Med. 2005; 353:2550-2558. [PubMed: 16354891]

49. Zigmond AS, Snaith RP. The hospital anxiety and depression scale. Acta Psychiatr Scand. 1983; 67:361-370. [PubMed: 6880820]

50. Snaith RP. The Hospital Anxiety And Depression Scale. Health Qual Life Outcomes. 2003; 1:29. [PubMed: 12914662] 


\section{Study Highlights}

\section{What is the current knowledge on the topic?}

Nonadherence to treatment in SLE varies widely depending on the study and assessment method used. Frequency of nonadherence in SLE patients with flares, who are thereby candidates for treatment escalation or may enter pharmaceutical trials, is unknown.

\section{What question did this study address?}

For the first time, we evaluated nonadherence to HCQ treatment in SLE patients with flares using drug levels and self-questionnaires.

\section{What this study adds to our knowledge}

Drug levels objectively identified that 1 patient in 5 was severely nonadherent, often without the physician's knowledge.

HCQ levels and self-administered questionnaires measured two different patterns of nonadherence: questionnaires mostly captured relatively infrequently missed tablets, while very low blood drug levels identified complete interruption or erratic tablet intake.

\section{O How this might change clinical pharmacology or translational science}

The frequency with which physicians miss nonadherence, together with under-reporting by patients, suggest that therapeutic drug monitoring is useful in real life and should be a prerequisite to inclusion in pharmaceutical trials. 


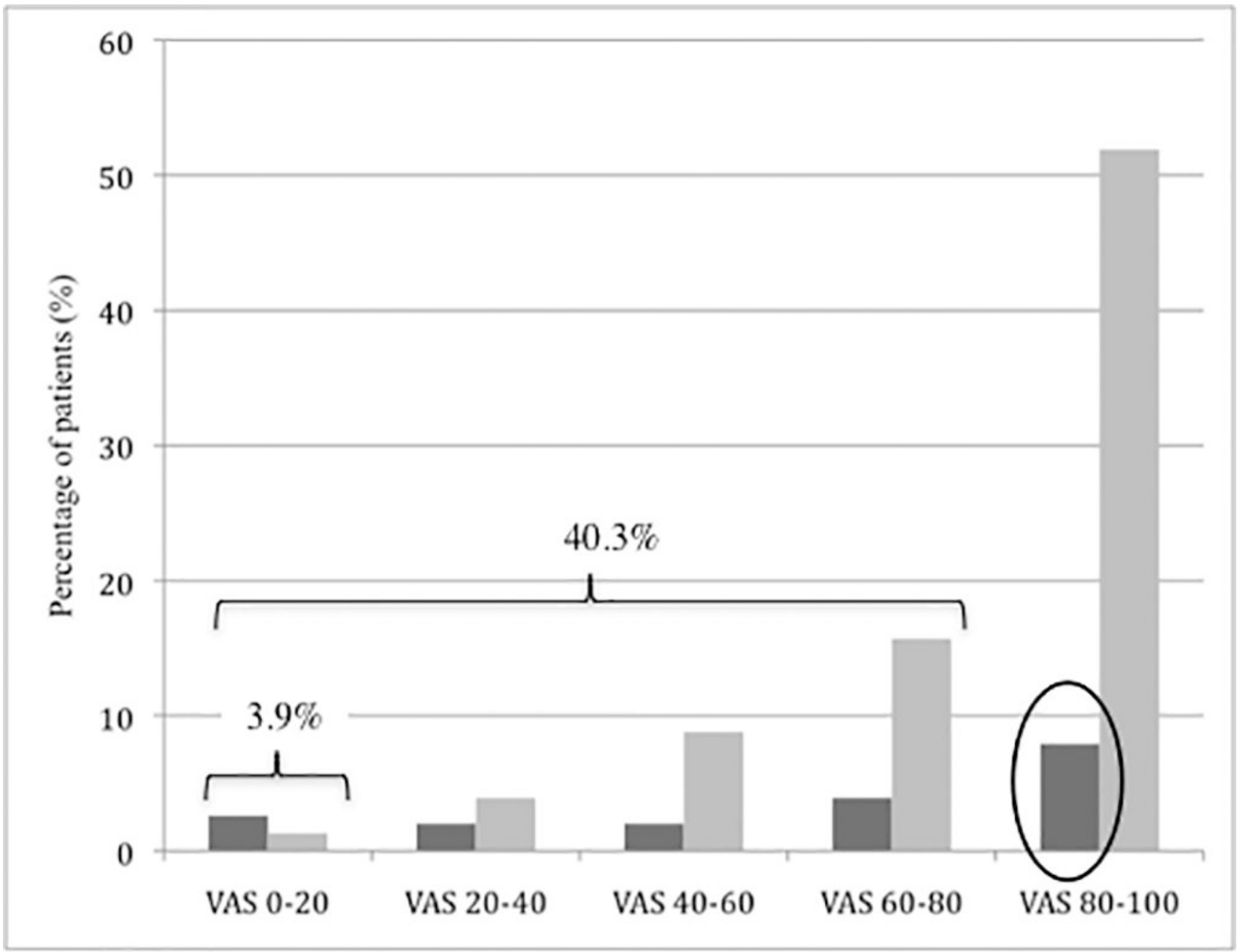

Figure 1. Adherence as estimated by physicians

In the histograms, the dark gray rectangles represent nonadherent patients by drug levels, and the light gray rectangles the others.

The patient's physician scored all components of the flare composite index and estimated adherence to HCQ treatment in the past month on a VAS ranging from 0 (patient took no treatment) to 100 (patient took all treatment). Physicians estimated that 123/305 patients $(40.3 \%)$ took less than $80 \%$ of their HCQ treatment in the previous month and that only $12 / 305(3.9 \%)$ took less than $20 \%$ of it. Physicians considered many of the nonadherent patients by drug levels to be adherent (circle). 


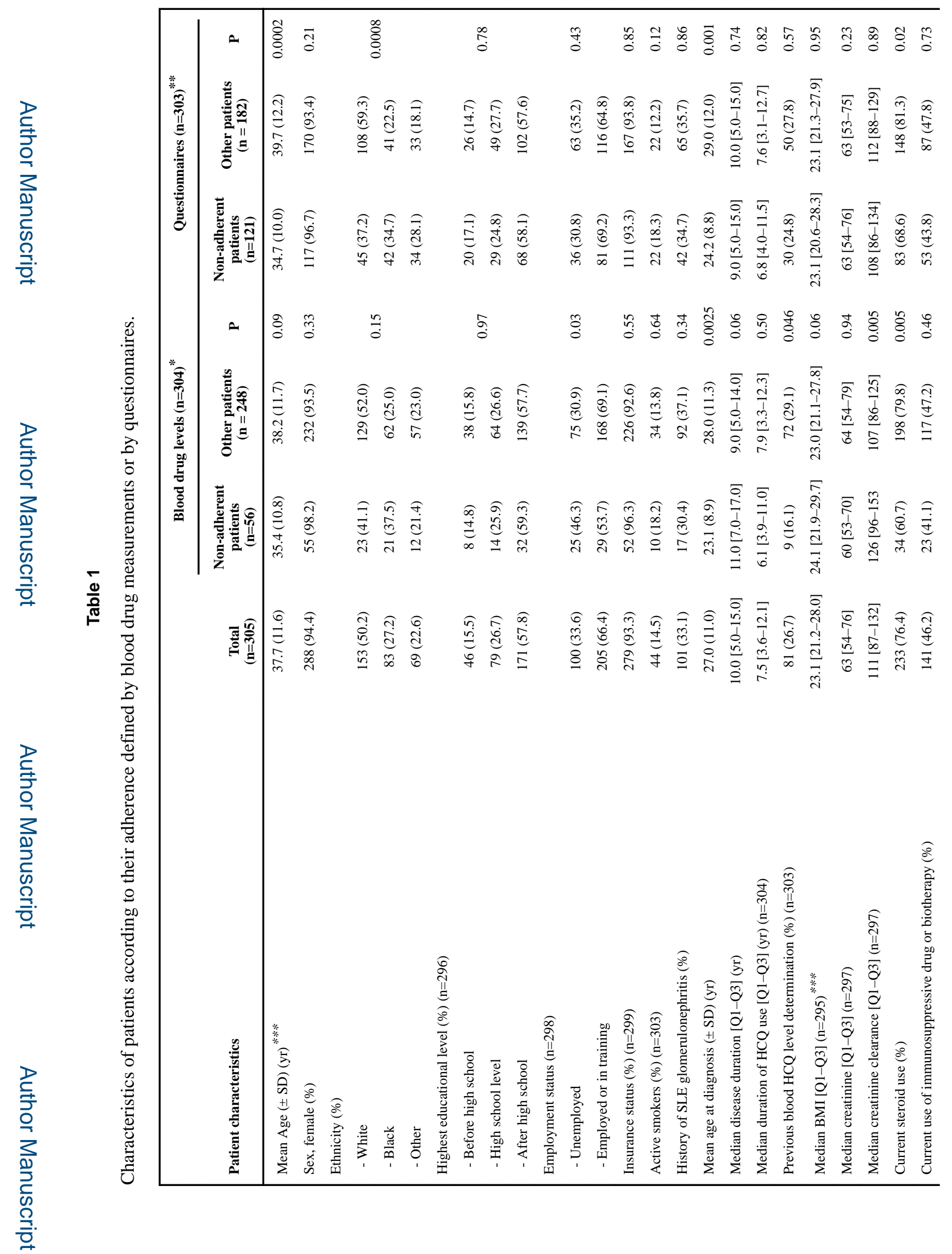

Clin Pharmacol Ther. Author manuscript; available in PMC 2019 June 01. 


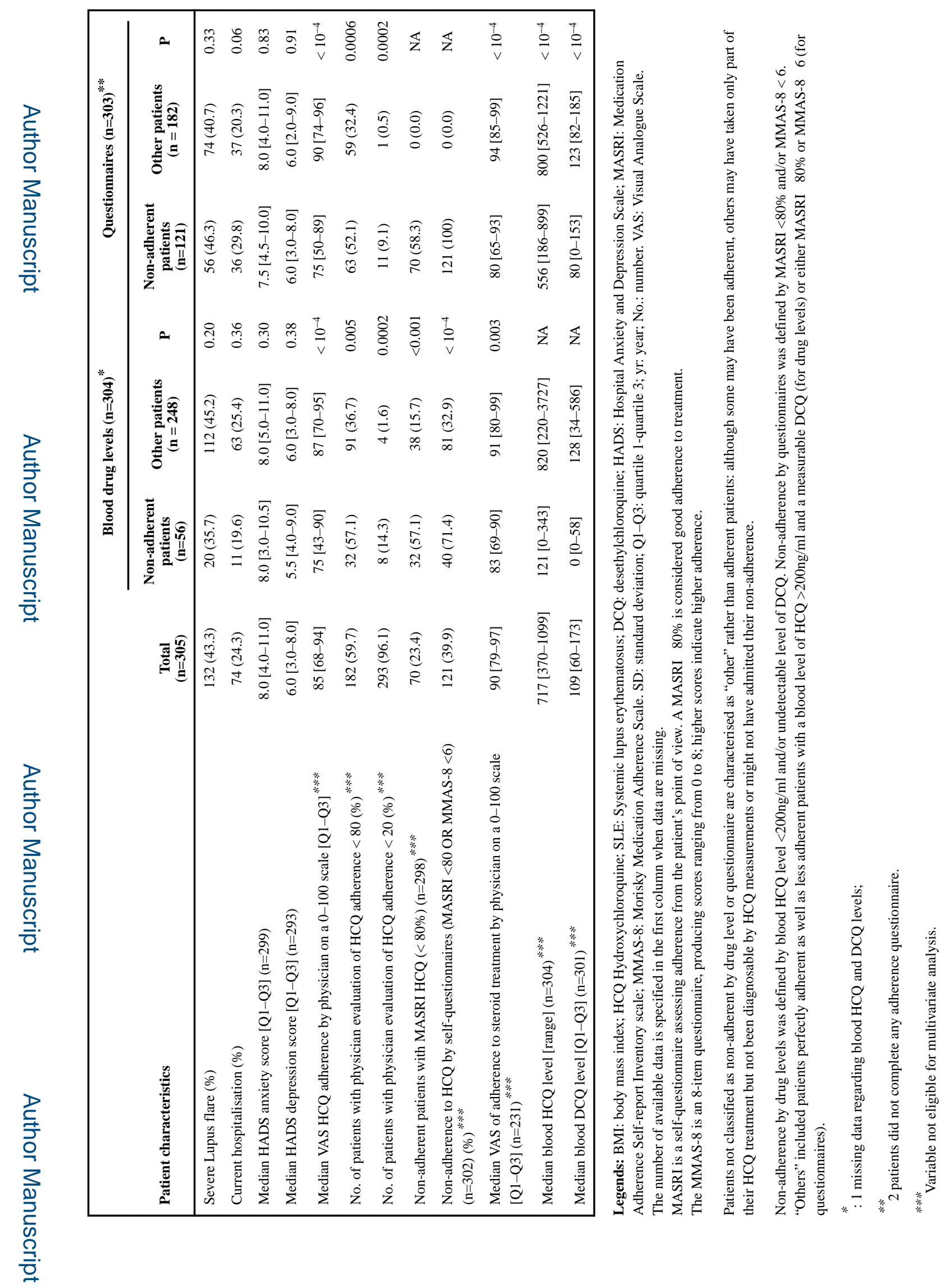

Clin Pharmacol Ther. Author manuscript; available in PMC 2019 June 01. 


\section{Table 2}

Multivariate analysis of predictors of non-adherence defined by blood drug measurements.

\begin{tabular}{lccc}
\hline & Odds ratio & $\begin{array}{c}\mathbf{9 5 \%} \text { confidence } \\
\text { interval }\end{array}$ & $\mathbf{p}$ \\
\hline Younger age at diagnosis (per 5 years) & 0.71 & $0.59-0.85$ & $<\mathbf{0 . 0 0 1}$ \\
Absence of steroids & 3.73 & $1.84-7.56$ & $<\mathbf{0 . 0 0 1}$ \\
Higher BMI (per $\left.\mathbf{5} \mathbf{~ k g / \mathbf { m } ^ { 2 }}\right)$ & 1.35 & $1.06-1.72$ & $\mathbf{0 . 0 1 7}$ \\
Being unemployed & 2.38 & $1.23-4.61$ & $\mathbf{0 . 0 1 0}$ \\
\hline
\end{tabular}

Legends: BMI: body mass index. 
Table 3

Multivariate analysis of predictors of non-adherence defined by questionnaires.

\begin{tabular}{lccc}
\hline & Odds ratio & $\begin{array}{c}\text { 95\% confidence } \\
\text { interval }\end{array}$ & $\mathbf{p}$ \\
\hline Age at diagnosis (per 5 years) & 0.79 & $0.70-0.90$ & $<\mathbf{0 . 0 0 1}$ \\
Absence of steroids & 2.29 & $1.28-4.10$ & $\mathbf{0 . 0 0 5}$ \\
Ethnicity & & & \\
$\quad$ Black vs white & 2.03 & $1.15-3.61$ & $\mathbf{0 . 0 1 5}$ \\
Other vs white & 2.19 & $1.19-4.06$ & $\mathbf{0 . 0 1 2}$ \\
\hline
\end{tabular}




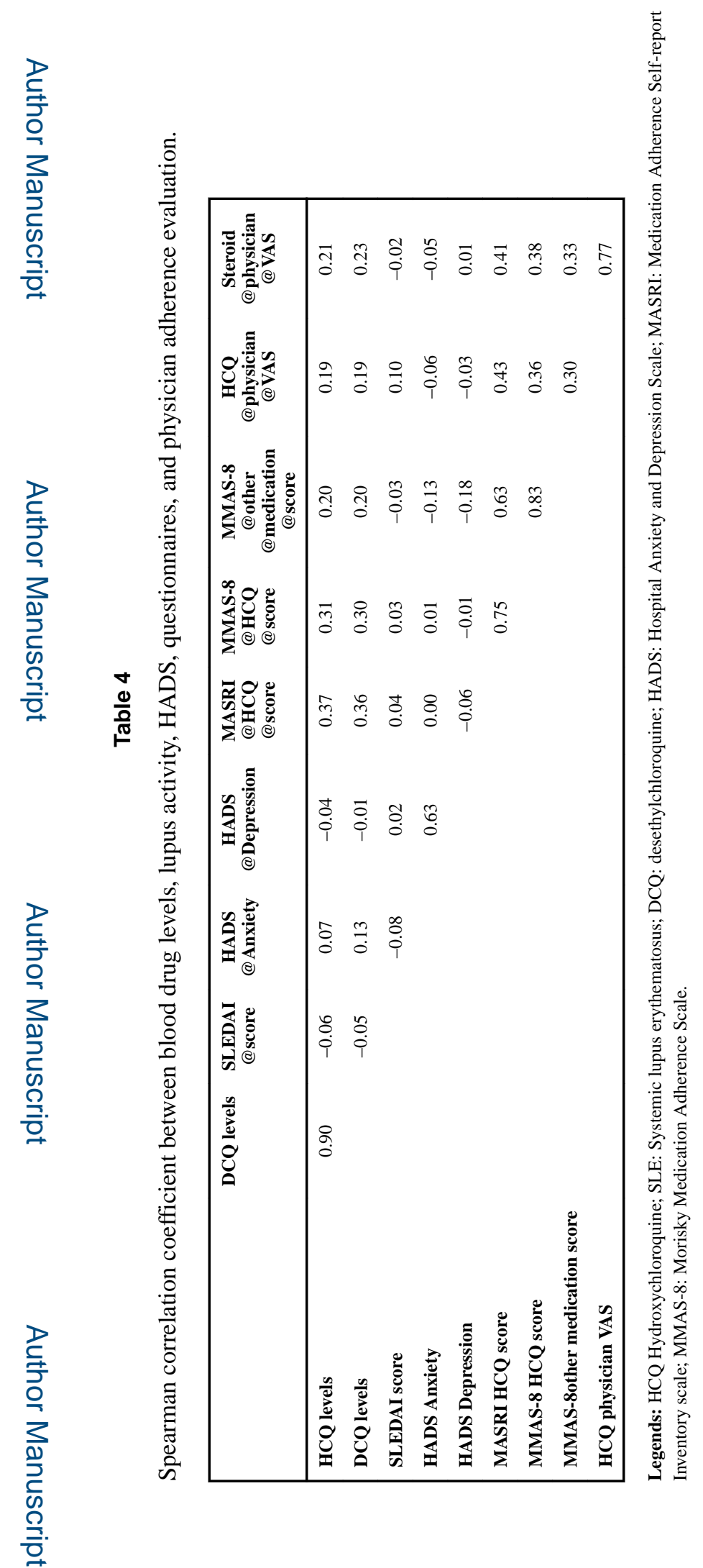

Clin Pharmacol Ther. Author manuscript; available in PMC 2019 June 01. 